Cahiers
de la Recherche
sur les Droits

Cahiers de la recherche sur les droits

Fondamentaux fondamentaux

$14 \mid 2016$

Urbanisme et droits fondamentaux

\title{
Urbanisme et discrimination
}

Town-Planning and Discrimination

\section{François Priet}

\section{OpenEdition}

Journals

Édition électronique

URL : https://journals.openedition.org/crdf/581

DOI : $10.4000 /$ crdf.581

ISSN : 2264-1246

Éditeur

Presses universitaires de Caen

Édition imprimée

Date de publication : 1 novembre 2016

Pagination : 11-19

ISBN : 978-2-84133-838-2

ISSN : 1634-8842

Référence électronique

François Priet, "Urbanisme et discrimination », Cahiers de la recherche sur les droits fondamentaux [En ligne], 14 | 2016, mis en ligne le 01 octobre 2019, consulté le 14 novembre 2022. URL : http:// journals.openedition.org/crdf/581; DOI : https://doi.org/10.4000/crdf.581 


\title{
Urbanisme et discrimination
}

\author{
François PRIET \\ Professeur de droit public à l'université d'Orléans \\ Centre de recherche juridique Pothier (CRJ, EA 1212) \\ Directeur du Groupement de recherche sur les institutions et le droit de l'aménagement, de l'urbanisme et de l'habitat (GRIDAUH)
}

\section{Discrimination et méthode des politiques urbaines}

A. Un outil au service des politiques urbaines

B. La discrimination: de la sophistication à l'impuissance?

\section{Urbanisme et traitement discriminatoire}

A. L'utilisation dévoyée des procédures d'urbanisme

B. Vers des discriminations à rebours?

Envisagée en tant que «traitement différencié, inégalitaire, appliqué à des personnes sur la base de critères variables $»^{1}$, la notion de discrimination est en quelque sorte l'hydre que toute pensée juridique entend combattre, du moins en principe ${ }^{2}$. Les juristes représentant toutes les branches du droit sont enrôlés dans ce combat, qu'il s'agisse du spécialiste de droit de la consommation, du travailliste réfléchissant sur le contenu du contrat de travail ou, last but not least, de l'administrativiste qui traque les discriminations que le fonctionnement d'un service public serait susceptible d'engendrer vis-à-vis des usagers. Au cour de la discrimination, il y a l'égalité méconnue. Mais la notion sous-entend que toute violation du principe d'égalité ne constitue pas à elle seule une discrimination; encore faut-il que la décision entachée de discrimination s'appuie, de manière médiate ou sournoise, sur des critères liés à l'origine, au sexe, ou à la religion.

Cette approche péjorative n'est en réalité qu'un simple glissement du sens premier de la notion.

Un tel sens est ancien puisqu'on le trouve très clairement chez Cicéron ${ }^{3}$ où le terme se rattache à une opération intellectuelle visant à distinguer, discerner, ce qui entraîne nécessairement l'idée de classement, de comparaison, de hiérarchie entre différents éléments. Au XIX siècle, Littré signale l'utilisation du mot dans le domaine de la psychologie, et au XX $\mathrm{XX}^{\mathrm{e}}$ siècle la philosophie en fait un large usage. C'est donc sans surprise que, au sein des sciences sociales, les juristes le font leur, et le Vocabulaire juridique

1. Trésor de la langue française informatisé, entrée «Discrimination", en ligne: http://www.cnrtl.fr/definition/discrimination.

2. Il est évidemment inutile d'insister sur le fait que le droit peut aussi être au service de la discrimination, comme l'a montré le Code noir (1685) dont l'article 28 disposait: «Déclarons les esclaves ne pouvoir rien avoir qui ne soit à leurs maîtres; et tout ce qui leur vient par industrie, ou par la libéralité d'autres personnes, ou autrement, à quelque titre que ce soit, être acquis en pleine propriété à leurs maîtres, sans que les enfants des esclaves, leurs pères et mères, leurs parents et tous autres y puissent rien prétendre par successions, dispositions entre vifs ou à cause de mort; lesquelles dispositions nous déclarons nulles, ensemble toutes les promesses et obligations qu'ils auraient faites, comme étant faites par gens incapables de disposer et contracter de leur chef».

3. «Non est [...] consilium in vulgo, non ratio, non discrimen» [ "Il n’y a dans la foule ni réflexion, ni raison, ni discernement»] (Pro Cn. Plancio oratio [Plaidoyer pour Cn. Plancius], IV). 
publié sous la direction de Gérard Cornu peut écrire que le sens étymologique du terme «conserve sa vocation légitime à caractériser, dans l'analyse juridique, l'action de distinguer avec justesse et acuité, ce qui ne renvoie ni à l'arbitraire ni même à la seule subtilité juridique mais à la capacité d'établir des distinctions exactes» ${ }^{4}$.

Dans ces conditions, la discrimination au sens premier apparaît au cœur de l'urbanisme, envisagé en tant que science de l'aménagement des villes ${ }^{5}$. L'urbanisme est une discipline créée pour ne pas subir les effets potentiellement désastreux pour la santé et la sécurité publiques, les espaces naturels et l'esthétique, d'une urbanisation incontrôlée, favorisée par le développement économique, les besoins en logements et la célérité croissante des techniques de construction. Pour conjurer le chaos urbain, le droit applicable à l'urbanisme doit dans un premier temps s'efforcer de distinguer entre les différentes portions de l'espace d'une ville ou d'une agglomération, déterminer leurs caractéristiques physiques et démographiques actuelles, leurs potentialités de transformation. L'opération de discrimination est bien à la base du travail de l'urbaniste, puis du juriste et de l'administrateur appelés à en tirer les conséquences (I).

Cet aspect technique de la discrimination, presque intrinsèquement vertueux, est pourtant occulté par un versant beaucoup plus sombre, qui ramène au sens péjoratif de la discrimination. L'utilisation des règles d'urbanisme par l'Administration, d'État jadis, locale à présent, n'a pas toujours bonne presse. Le trait de l'urbaniste, figé par la planification urbaine, peut faire la fortune des uns et le malheur des autres, sans que les justifications apparaissent toujours avec évidence, et ce malgré toutes les procédures de participation du public que le législateur a multipliées depuis près d'une trentaine d'années, souvent pressé par le droit de l'Union européenne (II).

Si ces deux faces de la discrimination dans le domaine de l'urbanisme doivent être distinguées, il peut arriver que l'on passe insensiblement de la discrimination-procédé au traitement discriminatoire. En outre, la discrimination, quel que soit son sens, est parfois le révélateur d'une certaine crise de l'encadrement des politiques urbaines par le droit.

\section{Discrimination et méthode des politiques urbaines}

Le principe même de la planification urbaine consiste à différencier l'espace entre différentes affectations, garanties par un vaste ensemble de servitudes administratives qui, à la différence des servitudes destinées à protéger le domaine public, ont vocation à grever l'ensemble des propriétés. La discrimination est donc à la base même du travail du planificateur. En tant qu'outil au service des politiques urbaines, elle n'a cessé de se perfectionner (A), pour trouver peut-être aujourd'hui ses limites (B).

\section{A. Un outil au service des politiques urbaines}

Discriminer l'espace pour introduire la rationalité rendue nécessaire par le développement des villes, telle était dès l'origine l'intention des auteurs de la loi du 14 mars 1919 concernant les plans d'extension et d'aménagement des villes. C'est pourtant dans une certaine discrétion que la technique apparaîtra, avant de connaître l'expansion qu'on lui connaît aujourd'hui. Le juge administratif sera amené à lui conférer une grande portée.

1. Loin de la prolixité des textes actuels, la loi de 1919 fixe le contenu des plans d'aménagement, d'embellissement et d'extension en le réduisant à quelques éléments essentiels. En particulier chaque plan doit établir un programme « déterminant les servitudes hygiéniques, archéologiques et esthétiques ainsi que toutes les autres conditions y relatives $[\ldots]$ ». L'idée de spécialisation de l'espace figure déjà dans la loi mais ce n'est que dans des textes ultérieurs que la possibilité de créer des zones réservées à un certain usage est expressément mentionnée (loi du 19 juillet 1924 complétant la loi du 14 mars 1919, loi du 14 mai 1932 autorisant l'établissement d'un projet d'aménagement de la région parisienne).

C'est la raison pour laquelle le Conseil d'État statuant en assemblée dans une décision Lainé du 23 février 1934 a pu considérer qu'il résultait de l'ensemble des dispositions de la loi du 14 mars 1919 (telle sans doute qu'elle qu'avait été modifiée par la loi de 1924, les faits de l'espèce remontant à 1930) que les plans d'aménagement pouvaient «prévoir une répartition du territoire des villes [...] en zones grevées de servitudes particulières dans l'intérêt de l'hygiène, de l'archéologie et de l'esthétique ${ }^{6}$.

Dans son commentaire de la décision, son rapporteur, le président Laroque, souligne le lien nécessaire, nettement exprimé par la loi, entre le choix d'une zone déterminée et l'existence d'une préoccupation d'ordre hygiénique, archéologique ou esthétique. Si l'urbanisme est une police spéciale, son utilisation n'est légale que dans la mesure où le choix d'une zone satisfait à tout ou partie des intérêts publics définis par la loi, quelle que soit leur nature. La notion de circonstances locales, bien connue dans le domaine de la police générale, est simplement adaptée aux buts particuliers de la police de l'urbanisme.

Mais à peine ce blanc-seing est-il accordé aux autorités locales que surgit la question des limites de la discrimination susceptible d'être pratiquée entre les différents secteurs d'un

4. Vocabulaire juridique, $7^{\mathrm{e}}$ éd., G. Cornu (dir.), Paris, PUF, 2005, entrée «Discrimination».

5. S'il y a désormais bien longtemps que le droit applicable à l'urbanisme prend aussi en compte les espaces ruraux, c'est toujours en tant qu'un processus d'urbanisation est en cause, d'un point de vue actif ou passif.

6. CE, Ass., 23 février 1934, Lainé, Recueil Lebon, p. 267; Recueil Sirey, 1935, 3, 9, note P. Laroque. 
territoire urbain. C'est tout l'enjeu des développements des pouvoirs de l'Administration que perçoit de manière visionnaire le président Laroque dans sa note. Déjà l'arrêt Lainé admet qu'un plan puisse distinguer, au sein des zones réservées à l'habitation, deux sous-catégories, les «zones d'habitation collective», et les «zones résidentielles ", dès lors que, en l'espèce, des considérations d'hygiène et d'esthétique le justifient. Le président Laroque montre parfaitement que c'est seulement à l'aune des critères prévus par la loi à l'époque applicable que les auteurs d'un plan peuvent délimiter des zones autres que celles réservées à l'habitation - la réponse est clairement négative pour la création de zones agricoles -, ou, à l'intérieur des zones réservées à l'habitation, établir des sous-distinctions, suivant que les zones en question admettent également l'industrie et le commerce, ou les excluent, ou encore se contentent de les restreindre.

Le raisonnement du président Laroque repose néanmoins sur un présupposé: celui selon lequel les motifs de délimitation des zones bénéficient d'une certaine stabilité. Or, l'évolution de la croissance urbaine, ainsi que les conceptions des politiques d'aménagement, vont conduire à étendre fortement les préoccupations qui doivent être celles des collectivités publiques en matière d'urbanisme, de telle sorte que les considérations tirées de «l'hygiène, de l'archéologie et de l'esthétique» vont apparaître beaucoup trop réductrices. Dès l'acte dit loi du 15 juin 1943, la loi officialise la technique du zonage et lui donne une étendue que les nombreuses réformes qui suivront ne feront qu'amplifier.

2. Face à cette extension des pouvoirs de l'Administration, le juge montre à l'origine qu'il entend contrôler assez strictement la délimitation des zones, en tant qu'elles constituent des atteintes au droit de propriété. Telle est tout au moins la lecture que l'on peut faire de l'arrêt Lainé précité, là encore sous le bénéfice des observations du président Laroque. C'est bien un contrôle normal de qualification juridique que la haute assemblée effectue ici. L'annotateur croit être en mesure de prédire, si on le lit bien, que le Conseil d'État ne sera pas loin d'être un juge administrateur: "rien ne montre mieux à quel point le juge administratif est mêlé à l'administration active, et la profondeur de l'action qu'il exerce sur elle». Une certaine figure du juge se dessine ici : celle d'un juge qui saura accompagner l'Administration dans sa tâche de planification, en censurant si nécessaire les zonages inadéquats.

En vérité, qu'il ne l'ait pas pu ou voulu, le juge n'a guère joué le rôle qu'on pouvait attendre de lui, ce retrait étant le résultat d'une combinaison de facteurs dont il est assez difficile de déterminer l'importance respective.

L'évolution des textes a étendu sensiblement pour ne pas dire démesurément la faculté ouverte à l'Administration de répartir le territoire des villes entre différentes zones, au fur et à mesure de l'extension des préoccupations du droit de l'urbanisme: en particulier la protection de l'agriculture, dans le cadre des zones dites NC (terminologie des plans d'occupation des sols, POS) ou A (terminologie des plans locaux d'urbanisme, PLU), et la sauvegarde des espaces naturels (zones ND des POS, ou $\mathrm{N}$ des $\mathrm{PLU}$ ). Plus généralement encore, l'accroissement et la diversification des grands intérêts publics que les auteurs des documents d'urbanisme doivent prendre en compte, en recourant aux délicats arbitrages qui en découlent, conduisent mécaniquement à complexifier la technique du zonage: discriminer l'espace, c'est fixer des réglementations particulièrement fines au sein de sous-secteurs pour lesquels le Code de l'urbanisme laisse, au demeurant, toute liberté aux autorités locales.

Le juge administratif a alors préféré se limiter à un contrôle de l'erreur manifeste dans le classement des terrains entre les différentes catégories de zones prévues par le Code. Depuis l'arrêt Commune de Bouchemaine ${ }^{7}$, éclairé par les conclusions de Daniel Labetoulle, le Conseil d'État estime qu'il ne lui appartient pas de contrôler la qualification juridique des faits, autrement dit de déterminer si les caractéristiques d'un secteur répondent à la définition des zones telle qu'elle est fixée par les dispositions législatives et réglementaires. Le juge aurait pu le cas échéant contrôler l'adéquation entre l'édiction d'une règle d'urbanisme et l'objectif voulu par ses auteurs, tel qu'il est (tel qu'il doit être) justifié par le rapport de présentation du plan d'urbanisme : il a préféré, ici aussi, se limiter au contrôle de l'erreur manifeste ${ }^{8}$.

Dans ces conditions, la discrimination entre les différents secteurs du territoire communal, en tant que technique trouvant directement sa source dans la loi, rend sans objet le principe d'égalité, ainsi que le Conseil d'État l'a rappelé à plusieurs reprises: seule l'existence d'une erreur manifeste dans le choix d'un zonage pourrait par ricochet attester de l'existence d'une méconnaissance du principe d'égalité ${ }^{9}$. Cette liaison présentée comme logique par la haute assemblée entre le contrôle restreint des motifs de fait et le contrôle de la violation de la loi n'emporte pas nécessairement la conviction: on peut penser que, dans certaines circonstances, il pourrait y avoir place pour un contrôle propre du respect du principe d'égalité. Dans le domaine de la planification urbaine, la loi n'écarte le principe que dans la mesure où il s'agit

7. CE, sect., 23 mars 1979, Commune de Bouchemaine, Recueil Lebon, p. 127, concl. D. Labetoulle.

8. CE, 29 décembre 1993, Société de Torrès, Recueil Lebon, tables, p. 1090: Bulletin de jurisprudence de droit de l'urbanisme, no 3, 1994, p. 85, concl. S. Fratacci.

9. CE, 3 novembre 1982, $M^{\text {lle }}$ Bonnaire, Recueil Lebon, p. 363: "Cons. qu'il est de la nature de toute réglementation d'urbanisme de distinguer des zones où les possibilités de construire sont différentes, ainsi que des zones inconstructibles; que, dès lors que cette délimitation ne repose pas, ainsi qu'il a été dit ci-dessus, sur une appréciation manifestement erronée, elle ne porte pas d'atteinte illégale au principe d'égalité des citoyens devant la loi». 
pour l'autorité compétente de prévoir des réglementations différentes afin de satisfaire des objectifs d'urbanisme. À partir du moment où la situation d'un secteur n'est pas fondamentalement différente d'un autre, et dans la mesure évidemment où l'Administration, dans le cadre du débat contentieux, n'apporte pas d'éléments de preuve significatifs, la sanction du principe d'égalité pourrait redevenir un moyen de contrôle autonome ${ }^{10}$. On peut d'autant plus le souhaiter que le principe de non-indemnisation des servitudes d'urbanisme, bénéficiant désormais d'un brevet de constitutionnalité ${ }^{11}$, ferme en quelque sorte le piège dans lequel le droit positif enferme les propriétaires: l'approfondissement du contrôle du juge permettrait de conférer au principe de non-indemnisation la légitimité que tant d'auteurs et de plaideurs lui dénient depuis plus de quatre-vingts ans.

Dans ses conclusions précitées, le président Labetoulle proposait une ouverture intéressante en invitant le cas échéant le Conseil d'État à vérifier que le choix d'un zonage ou d'une réglementation répondît bien à un «motif d'urbanisme». Il n'est pas sûr qu'un tel type de contrôle contribue significativement à limiter le pouvoir de discrimination de l'espace ouvert aux autorités administratives: ainsi, c'est parce qu'un tel motif existait en l'espèce que le Conseil d'État avait admis que le POS de Lourdes puisse limiter l'implantation de commerces de détail au-delà d'un certain seuil de surface commerciale: le monde de l'entreprise n'a sans doute guère apprécié cette immixtion du droit des sols dans ses activités ${ }^{12}$. En définitive, le «motif d'urbanisme», par sa plasticité même, renforce plus qu'il ne le bride le pouvoir de l'Administration.

Toujours est-il qu'un tel contrôle oblige l'Administration à s'expliquer, et le récent décret $\mathrm{n}^{\circ} 2015-1783$ du 28 décembre 2015 insiste fortement sur la fonction explicative du rapport de présentation ${ }^{13}$. C'est à ce prix que la discrimination légale à laquelle procède le droit de l'urbanisme est acceptable par le citoyen.

\section{B. La discrimination: de la sophistication à l'impuissance?}

Ce n'est pas la méthode de la discrimination de l'espace en tant que telle qui fait débat aujourd'hui, c'est sans doute l'excès de réglementation contenue dans le règlement des différentes zones.
1. Si ce ne sont pas les seuls facteurs d'explication, les exigences de la prise en compte de l'environnement et la nécessité de favoriser la production de logements dans un objectif de mixité sociale au sein des quartiers conduisent à sophistiquer à l'extrême la réglementation d'urbanisme. $\mathrm{Au}$-delà des règles particulières, de plus en plus précises, que les auteurs du règlement de PLU peuvent édicter, deux mécanismes contribuent à la mise en œuvre de ces objectifs : la sectorisation et la dérogation.

La sectorisation se présente comme un sous-produit dérivé de la technique du zonage, afin de répondre aux enjeux que le législateur, depuis une quinzaine d'années, impose aux élus locaux. C'est la raison pour laquelle la loi a multiplié les dispositions pour renforcer, au sein des différentes catégories de zones existantes, la spécialisation de l'espace, en permettant au règlement du plan de fixer dans certains secteurs des règles spécifiques en fonction des objectifs d'intérêt général recherchés. Dans le domaine de la protection de l'environnement tout d'abord, le procédé de la sectorisation est utilisé pour fixer des performances énergétiques et environnementales renforcées applicables aux constructions, travaux, installations et aménagements situés dans les secteurs concernés, en imposant le cas échéant une production minimale d'énergie renouvelable (art. L. 151-21 du Code de l'urbanisme). Il peut l'être également pour favoriser un regroupement des constructions afin de protéger la qualité paysagère de certaines zones (art. L. 151-25) ou pour favoriser les constructions faisant preuve d'exemplarité énergétique ou environnementale ou à énergie positive (art. L. 151-28 $3^{\circ}$ : les règles relatives au gabarit pourront être dépassées dans certaines limites). Ce procédé est plus encore utilisé lorsqu'il s'agit de répondre aux besoins en logements et de lutter contre l'étalement urbain, les deux objectifs ne pouvant pas, en tout état de cause, être poursuivis indépendamment l'un de l'autre. Deux tendances se dégagent ici. Soit le droit positif favorise l'hyperspécialisation lorsqu'il autorise le règlement du plan à imposer une densité maximale de constructions dans des secteurs qu'il délimite au sein des secteurs euxmêmes situés à proximité des transports collectifs: c'est en quelque sorte la sectorisation dans la sectorisation (art. L. 151-26). Soit la loi permet au règlement du PLU de fixer des secteurs dans lesquels certaines règles du plan (gabarit, hauteur, emprise au sol) pourront être dépassées, dans certaines limites. Les conditions de dépassement sont elles-mêmes différentes selon qu'il s'agit de permettre

10. Les faits ayant donné lieu à la décision Laurent rendue par le Conseil d'État le 25 septembre 1987 (modestement publiée aux tables du Recueil Lebon, p. 1007) montrent les potentialités d'un tel contrôle: le requérant reprochait notamment aux auteurs du POS d'avoir classé en zone NC des terrains lui appartenant, alors que d'autres terrains présentant des caractéristiques comparables, mais situés à l'ouest du bourg, avaient reçu un classement autorisant des habitations isolées: l'absence d'erreur manifeste suffit à rejeter la requête sans que le juge pousse ses investigations pour déterminer dans quelle mesure le principe d'égalité a été respecté.

11. CE, 16 juillet 2010, SCI La Saulaie, Recueil Lebon, p. 315: Bulletin de jurisprudence de droit de l'urbanisme, nº 6, 2010, p. 460, concl. J. Boucher, obs. J. T.

12. CE, 7 mai 1986, Société Guyenne et Gascogne, n 57902: L'actualité juridique. Droit administratif, 1986, p. 523, note F. Bouyssou. Dans sa note précitée sous l'arrêt Lainé, le président Laroque avait cru pouvoir écrire à l'époque: «Il ne serait pas possible [...] d'établir, entre les différentes catégories d'industries ou de commerces, des distinctions fondées sur d'autres considérations, telles que la commodité des habitants. L'on ne saurait ainsi admettre une distinction entre le commerce de gros et le commerce de détail, tendant à autoriser celui-ci seul dans les zones réservées à l'habitation ".

13. Art. R. 151-2 du Code de l'urbanisme. 
l'agrandissement ou la construction de bâtiments à usage d'habitation, de prévoir des programmes de logements comportant des logements locatifs sociaux, ou des logements dits intermédiaires (art. L. $151-281^{\circ}, 2^{\circ}$ et $4^{\circ}$ ).

Second procédé, la dérogation a fait depuis quelques années un retour en force spectaculaire dans le droit de l'aménagement. Les deux objectifs précités justifient le recours à cette technique dans de nombreuses hypothèses. C'est au nom de la lutte contre le changement climatique que la loi habilite l'Administration à déroger aux règles relatives à l'aspect extérieur des constructions lorsqu'est en cause l'utilisation de matériaux renouvelables ou de matériaux ou procédés de construction permettant d'éviter l'émission de gaz à effet de serre, l'installation de dispositifs favorisant la retenue des eaux pluviales ou la production d'énergie renouvelable (art. L. 111-16). Afin de favoriser les économies d'énergie, l'autorité compétente pour délivrer les autorisations d'urbanisme peut déroger aux règles du plan relatives à l'emprise au sol, à la hauteur, à l'implantation et à l'aspect extérieur des constructions lorsque sont en cause certains travaux d'isolation ou de protection contre le rayonnement solaire (art. L. 152-5). L'objectif de construction de logements dans les «zones tendues", c'est-à-dire celles qui sont caractérisées par un déséquilibre marqué entre l'offre et la demande de logements ${ }^{14}$, a conduit le législateur à autoriser l'Administration à déroger à de nombreuses règles fixées par le plan (gabarit, densité, création d'aires de stationnement, implantation par rapport aux limites séparatives) pour permettre de construire, reconstruire, rénover ou réhabiliter des constructions à usage d'habitation (art. L. 152-6).

On peut donc constater que, à l'intérieur d'une même zone, les administrés seront géographiquement susceptibles d'être assujettis à des règles spécifiques, ou que, au contraire, l'application des règles sera assouplie par des systèmes variés de dérogations. La différenciation peut même avoir un caractère beaucoup plus général puisque le cas particulier des zones tendues aboutit à créer, sur une partie du territoire national, une sorte de droit de l'urbanisme à deux vitesses ${ }^{15}$.

2. L'application du principe d'égalité est prise dans une sorte de tourbillon: déjà malmené par la législation de l'urbanisme dès l'origine, le principe devient pratiquement insaisissable en raison de la différenciation presque à la carte que permet le droit positif; inversement, c'est la recherche du rétablissement de l'égalité qui explique certaines évolutions du droit positif. Ainsi, l'objectif de mixité sociale permet d'imposer que des programmes de logements comportent une proportion de logements d'une taille minimale, ou qu'un pourcentage de ces mêmes programmes soit affecté à des catégories de logements définies par le plan. La volonté de rendre accessibles les bâtiments aux personnes handicapées justifie la possibilité de déroger aux règles du PLU pour autoriser les travaux nécessaires.

Ces discriminations tous azimuts suscitent un certain malaise ${ }^{16}$ qui résulte sans doute de l'excès d'ambition du droit de l'urbanisme. Celui-ci n'est plus seulement une discipline qui cherche à établir un ordre urbain minimal, grâce à une distribution des différentes affectations de l'espace effectuée par un planificateur serein. Il doit désormais contribuer au bon fonctionnement de la ville. Les attentes du corps social, relayées par le législateur, sont telles que les autorités locales sont à présent tenues de répondre à toute la complexité du phénomène urbain. La notion de projet urbain, reprise du discours des urbanistes par le législateur, devient un projet de vie que les documents de planification urbaine ont pour mission de traduire, sachant que le projet propre à chaque ville doit s'inscrire dans les grands objectifs de vie en société. Les élus locaux doivent en quelque sorte prendre leur part dans la mise en œuvre du pacte républicain. Dans ces conditions, le Code de l'urbanisme constitue une boîte à outils dans laquelle les élus locaux sont invités à puiser pour mettre les services que peut offrir la ville - emplois, logements, équipements publics - à la disposition de tous. Autrement dit, la «bonne» discrimination - la faculté de distinguer - doit être utilisée pour combattre la mauvaise celle qui conduit au traitement discriminatoire.

On mesure à présent les effets pervers de la sophistication orchestrée depuis de nombreuses années par le législateur. La règle trop complexe, qui n'a plus que les apparences de la généralité, requiert de plus en plus souvent sa mise à l'écart par l'octroi de dérogations. Il peut même arriver que le législateur tempère la dérogation qu'il autorise par ailleurs : ainsi, tout en permettant à l'autorité compétente de déroger aux règles relatives à l'aspect extérieur des constructions pour permettre la mise en place de dispositifs écologiques, la loi l'habilite, comme par une sorte de remords, à assortir sa décision de «prescriptions destinées à assurer la bonne intégration architecturale du

14. Les «zones tendues» sont définies d’une manière générale par l’article 232 du Code général des impôts. Elles correspondent à des «communes appartenant à une zone d'urbanisation continue de plus de cinquante mille habitants où existe un déséquilibre marqué entre l'offre et la demande de logements, entraînant des difficultés sérieuses d'accès au logement sur l'ensemble du parc résidentiel existant, qui se caractérisent notamment par le niveau élevé des loyers, le niveau élevé des prix d'acquisition des logements anciens ou le nombre élevé de demandes de logement par rapport au nombre d'emménagements annuels dans le parc locatif social». La liste précise de ces communes est fixée par le décret n²013-392 du 10 mai 2013, modifié en dernier lieu par le décret nº 2015-1284 du 13 octobre 2015.

15. Il convient de souligner un effet collatéral de l'existence des zones tendues qui est d'ordre contentieux: depuis un décret n ${ }^{\circ} 2013-879$ du $1^{\text {er }}$ octobre 2013, les tribunaux administratifs sont compétents en premier et dernier ressort sur les recours concernant certaines autorisations d'urbanisme délivrées dans ces zones (permis de construire ou de démolir un bâtiment à usage principal d'habitation, permis d'aménager un lotissement). Il s'agit ici de réduire le temps du procès dans les secteurs où l'activité de construction est particulièrement importante pour satisfaire les besoins en logements. Cette disposition n'a toutefois qu'un caractère temporaire puisqu'elle s'applique aux recours introduits entre le $1^{\text {er }}$ décembre 2013 et le $1^{\text {er }}$ décembre 2018 .

16. Voire une critique radicale: S. Pérignon, «Pour en finir avec les urbanistes», Bulletin de jurisprudence de droit de l'urbanisme, $\mathrm{n}^{\circ} 3$, 2014, p. 163. 
projet dans le bâti existant et dans le milieu environnant » (art. L. 111-16 du Code de l'urbanisme) ${ }^{17}$. À tout le moins il faut souhaiter que le juge contrôle avec rigueur les dérogations susceptibles d'être octroyées à certaines règles fixées par les PLU dans les zones tendues: l'Administration peut les accorder «en tenant compte de la nature du projet et de la zone d'implantation dans un objectif de mixité sociale », ce qui ouvre de manière large et potentiellement inquiétante le champ de son pouvoir discrétionnaire... Il est vrai que le respect du principe d'égalité lorsqu'est en cause le pouvoir discrétionnaire de l'Administration est mieux assuré que par le passé ${ }^{18}$ : le juge accepte de vérifier, lorsque l'Administration a pris une certaine position dans des cas similaires, que sa décision ne méconnaît pas le principe d'égalité. En d'autres termes, dès lors que le pétitionnaire qui a essuyé un refus de dérogation apporte les précisions suffisantes sur sa situation par rapport à des précédents, l'Administration, si elle entend refuser la dérogation, doit convaincre le juge que son refus se justifie au regard d'un motif d'intérêt général, ou d'un motif propre à la situation du demandeur ${ }^{19}$. La cohérence de l'action administrative conditionne la légalité de l'exercice du pouvoir discrétionnaire.

La pratique du droit de l'urbanisme peut aussi susciter des traitements discriminatoires qu'il n'est pas toujours facile de combattre, ni même de connaître.

\section{Urbanisme et traitement discriminatoire}

Parmi les craintes que le transfert de la compétence urbanisme aux élus locaux suscitait figurait le risque de pratiques discriminatoires. Outre que l'accusation relève peut-être du procès d'intention, alors que l'urbanisme d'État était loin d'être sans reproches, il ne faut sans doute pas exagérer l'ampleur du phénomène. L'utilisation dévoyée des procédures d'urbanisme montre qu'il existe pourtant $(\mathrm{A})$. Un risque de « discrimination à rebours » n'est pas non plus à exclure (B).

\section{A. L'utilisation dévoyée des procédures d'urbanisme}

Si le droit de l'urbanisme n'a évidemment pas pour objet de définir le régime de la propriété, du moins l'influence-t-il de manière non négligeable. La tentation peut être grande d'utiliser certaines procédures dans un sens discriminatoire. Si des moyens existent pour combattre de telles pratiques, leur efficacité est très variable compte tenu de l’hétérogénéité des situations concernées.

1. C'est au premier chef aux personnes publiques compétentes que l'on peut imputer certaines utilisations dévoyées des procédures d'urbanisme; mais il convient de ne pas négliger un phénomène d'instrumentalisation de ces mêmes procédures lato sensu par les particuliers, dont le caractère discriminatoire est d'autant plus pernicieux qu'il est assez sournois.

Du côté des personnes publiques, la discrimination peut être alléguée en raison des classements tortueux relatifs à certaines parcelles opérés par les documents de planification urbaine. Il peut s'agir de classements effectués au détriment d'un propriétaire, ou au contraire à son avantage, soit à l'occasion d'une première élaboration d'un plan, soit plus couramment lors d'une procédure de modification ou de révision. Ce genre de pratiques se rencontrent peut-être plus fréquemment en zone rurale, en raison de la proximité entre les décideurs et les propriétaires, mais il serait naïf de considérer qu'un tel phénomène ne concerne pas aussi le milieu urbain. C'est sans doute un travers de la décentralisation telle qu'elle avait été conçue il y a plus de trente ans: le choix du niveau communal rendait très proche la relation entre les autorités locales et leurs administrés. Ce risque de liaisons dangereuses est appelé à s'estomper du fait de la généralisation du PLU intercommunal à la suite des dispositions de la loi ${ }^{\circ}{ }^{2010-}$ $788 \mathrm{du} 12$ juillet 2010 portant engagement national pour l'environnement (dite loi Grenelle II) et surtout de la loi $\mathrm{n}^{\circ}$ 2014-366 du 24 mars 2014 pour l'accès au logement et un urbanisme rénové (dite loi ALUR).

La planification urbaine n'est pas la seule procédure susceptible d'être à l'origine de discriminations puisque le droit de préemption urbain peut également être utilisé à des fins fort éloignées de l'intérêt général. La presse généraliste s'est fait l'écho de l'exercice du droit de préemption pour faire obstacle à des ventes de biens immobiliers à des personnes portant des noms à consonance étrangère, ou à des membres de la communauté des gens du voyage. Les conditions de légalité imposées par la jurisprudence administrative ne sont pas insurmontables, puisqu'il suffit que la décision de préemption soit justifiée par l'existence d'un projet $«$ réel $»^{20}$. Certes cela signifie bien que la collectivité locale ne peut pas s'appuyer sur une simple intention pour préempter légalement, mais il peut ne pas être très difficile, pour une mairie habile, de monter un projet qui aura toute l'apparence de la réalité pour échapper à la censure juridictionnelle.

17. Une même faculté est prévue dans le cas des dérogations mentionnées à l'article L. 152-5.

18. Le juge avait pu considérer que la circonstance que des dérogations semblables à celles accordées aient été refusées était sans influence sur la légalité de la décision (CE, sect., 16 décembre 1977, Ministre de l'équipement c. Cluzeau, Recueil Lebon, p. 509: L'actualité juridique. Droit administratif, 1978, p. 456, concl. M.-A. Latournerie).

19. CE, sect., 30 décembre 2010, Ministre du Logement et de la Ville c. M ${ }^{m e}$ Durozey, Recueil Lebon, p. 533, concl. G. Dumortier.

20. CE, 7 mars 2008, Commune de Meung-sur-Loire, Recueil Lebon, p. 97: Bulletin de jurisprudence de droit de l'urbanisme, $\mathrm{n}^{\circ}$ 1, 20o8, p. 57, concl. L. Derepas. 
Plus surprenante peut-être est l'affirmation selon laquelle les administrés eux-mêmes peuvent être à l'origine de comportements discriminatoires. Tel est pourtant le cas lorsque sont contestés des permis de construire portant sur des mosquées, des logements sociaux ou des établissements abritant des populations difficiles (prisons, centres d'accueil de jeunes en difficulté, etc.). Les requérants se transforment alors en gardiens sourcilleux du respect de certaines règles fixées par les plans d'urbanisme: bien souvent, les débats contentieux portent sur l'application de règles régissant la densité constructible, ou le nombre de places de stationnement, et qui concernent certains équipements qualifiés par le document d' $d$ équipements publics », d' "équipements collectifs », ou encore de "services d'intérêt collectif». La question est alors de savoir si le projet contesté peut être rattaché à ces notions telles que le règlement les définit ${ }^{21}$.

2. Face à de tels comportements, c'est dans un certain désordre que répond le droit positif: il est vrai que l'hétérogénéité des situations rend peu envisageables des solutions de caractère général.

Les classements douteux de parcelles peuvent donner prise au moyen du détournement de pouvoir. Mais ce moyen est aussi classique qu'il est peu efficace: en urbanisme comme dans d'autres domaines, la preuve est difficile à rapporter, et il n'est pas rare qu'un intérêt général relaie l'intérêt privé, absorbant ainsi ce dernier. Les dispositions du Code général des collectivités territoriales peuvent venir au secours des plaideurs. Est souvent invoqué à cet égard l'article L. 2131-11 qui déclare illégales les délibérations auxquelles ont pris part un ou plusieurs membres du conseil municipal intéressés à l'affaire qui en fait l'objet. Encore faut-il que la participation de l'élu ait eu une influence effective sur le sens de la délibération, le juge voulant éviter, en particulier dans les petites communes, une interprétation objective de la notion de «conseiller intéressé » qui fragiliserait bien des délibérations. Le fait d'être propriétaire foncier dans la commune ne suffit pas à exclure l'élu de sa participation au conseil municipal, ce qui aboutirait, au fond de manière assez paradoxale, à méconnaître le droit d'expression qu'une personne tient de sa qualité de conseiller municipal ${ }^{22}$.

Les recours contentieux à visée discriminatoire, drapés dans la défense de la légalité, peuvent être sérieusement encadrés par les règles qui régissent l'intérêt à agir. Jusqu'à la réforme issue de l'ordonnance no $2013-638$ du
18 juillet 2013, l'intérêt conférant qualité à agir contre une décision accordant un permis de construire reposait sur la notion de voisinage. La qualité de voisin du projet révélait en quelque sorte le caractère direct et personnel de l'intérêt à contester la décision. Cette qualité ne pouvait être que relative; elle dépendait de la nature et de la distance du lieu d'implantation du projet par rapport au requérant. C'est ainsi que le Conseil d'État a pu reconnaître l'intérêt à agir de personnes habitant à moins d'un kilomètre d'un projet de mosquée d'environ deux mille places ${ }^{23}$. Le resserrement des conditions de l'intérêt à agir par l'article L. 600-1-2 introduit par l'ordonnance précitée dans le Code de l'urbanisme est de nature à fortement limiter les recours de ce type, même si un tel resserrement a d'abord été voulu pour apporter une meilleure sécurité juridique et donc économique aux projets immobiliers. En exigeant, notamment, que la construction attaquée soit de nature à affecter directement les conditions d'occupation, d'utilisation ou de jouissance du bien que le requérant détient ou occupe régulièrement, la loi met fin à la présomption sur laquelle reposait l'intérêt à agir dans ce cas de figure. Le requérant devra convaincre le juge en lui fournissant «tous éléments suffisamment précis et étayés» de nature à justifier de l'atteinte qu'il invoque ${ }^{24}$. Le juge veille à ne pas transformer cette charge de la preuve en une probatio diabolica peu compatible avec le droit au recours: d'une part, le juge ne saurait aller jusqu'à exiger de l'auteur du recours qu'il apporte la preuve du caractère certain des atteintes qu'il invoque; d'autre part, le respect du caractère contradictoire de la procédure oblige à tenir compte des arguments contraires invoqués par l'administration défenderesse, le juge forgeant in fine sa conviction.

Enfin, le juge pénal peut-il sanctionner les discriminations auxquelles pourrait donner lieu l'exercice de certaines compétences d'urbanisme? Si aucun exemple ne peut semble-t-il être cité en matière de délivrance d'autorisations de construire, le seul cas que l'on peut évoquer concerne le droit de préemption urbain quand son exercice fait obstacle à un projet d'acquisition. Il est très décevant. L'article 432-7 du Code pénal sanctionne la discrimination commise à l'égard d'une personne physique ou morale commise par une personne dépositaire de l'autorité publique ou chargée d'une mission de service public, dans l'exercice ou à l'occasion de l'exercice de ses fonctions, lorsqu'elle consiste, notamment, "à refuser le bénéfice d'un droit accordé par la loi ». En dépit du caractère explicite des faits - un maire fait obstacle à une vente en utilisant son droit de préemption afin d'empêcher des personnes portant un nom

21. CE, sect., 12 février 1988, Association des résidents des quartiers Portugal-Italie, Recueil Lebon, p. 66: L'actualité juridique. Droit administratif, 1988, p. 293, concl. E. Guillaume (un centre culturel islamique est-il un «équipement public» : réponse affirmative); CAA Lyon, 6 mars 2012 , n 10 LYo2856 (une mosquée est-elle un "service d'intérêt collectif» : réponse affirmative); CE, 15 juin 2001, Commune de Vieux-Boucau, ${ }^{\circ}$ 218119: Construction - Urbanisme, novembre 2001, comm. n²29, note P. Cornille (un centre d'accueil et d’hébergement pour jeunes en difficulté est-il un «bâtiment scolaire, hospitalier ou sanitaire» : réponse négative). Le juge prend toujours soin d’indiquer que la notion est interprétée "au sens du POS (ou du PLU)».

22. CAA Douai, 8 septembre 2014, $\mathrm{n}^{\circ}$ 13DA00765: Revue de droit immobilier, 2014, p. 656, obs. P. Soler-Couteaux.

23. CE, 3 février 1992, $M^{\text {me }}$ Girod, Recueil Lebon, tables, p. 1396: solution implicite mais expressément fichée sur ce point.

24. CE, 10 juin 2015, M. Brodelle et $M^{m e}$ Gino, Recueil Lebon, p. 192 : Bulletin de jurisprudence de droit de l'urbanisme, $\mathrm{n}^{\circ} 5,2015, \mathrm{p} .368$, concl. A. Lallet, obs. J. T. 
à consonance étrangère de s'installer dans la commune -, la Cour de cassation n'a pu faire qu'une interprétation stricte de la loi pénale: d'une part, le droit de propriété, auquel fait obstacle la décision de préemption, ne saurait s'analyser comme un «droit accordé par la loi» dès lors que nul ne dispose d'un droit à devenir propriétaire; d'autre part, un tel droit trouve essentiellement sa source dans la manifestation de volonté du vendeur, et non pas dans la loi à proprement parler ${ }^{25}$. L'obstruction de l'élu ne présente qu'un caractère indirect au regard des prescriptions fulminées par la loi. Dès lors qu'il apparaît peu vraisemblable qu'une modification de l'incrimination soit décidée par le Parlement, le caractère discriminatoire d'une décision de préemption ne peut être sanctionné qu'indirectement par le juge administratif dans le cadre classique du recours pour excès de pouvoir, ce qui ne répond guère à la nature particulière du problème en cause.

Il reste à se demander si certaines évolutions ne seraient pas de nature à faire apparaître de nouvelles figures en matière de discrimination, ce que l'on pourrait appeler des «discriminations à rebours».

\section{B. Vers des discriminations à rebours?}

Il ne s'agit peut-être ici que de droit fiction, lié aux incidences de la jurisprudence de la Cour européenne des droits de l'homme lorsqu'elle fait application de l'article 8 de la Convention protégeant le droit de toute personne au respect notamment de sa vie privée et familiale, et de son domicile. Bien que la Convention européenne ne traite pas des questions d'urbanisme, la jurisprudence de la Cour sur la protection du domicile des gens du voyage conduit à certains frottements entre le droit humanitaire européen et notre droit public des sols.

1. Le législateur a mis en place un double mécanisme pour s'assurer du respect des règles d'urbanisme, en particulier celles qui sont fixées par les documents de planification: en amont, un grand nombre de travaux, aménagements et ouvrages ne peuvent être exécutés que s'ils ont été autorisés; en aval, l'absence d'autorisation, ou le fait de ne pas réaliser les travaux conformément à l'autorisation accordée, sont pénalement sanctionnés. C'est peu dire que l'installation des gens du voyage fait mauvais ménage avec ce cadre juridique. Pourtant la Cour européenne des droits de l'homme oblige les États parties à la Convention à concilier le respect des règles d'urbanisme et celui du droit au domicile, domicile dont la Cour estime qu'il convient d'en donner une définition utile au regard de l'objectif de la Convention: le domicile ne peut pas être seulement le lieu de vie protégé par un titre juridique, mais il doit être reconnu comme tel dès lors qu'existent des liens suffisants et continus avec un lieu déterminé. Au demeurant la Cour a tendance à lier respect du domicile et respect de la vie privée et familiale ${ }^{26}$.

Si une réglementation d'urbanisme constitue bien une ingérence d'une autorité publique dans l'exercice d'un tel droit, cette ingérence ne peut être compatible avec la Convention que si, selon son article 8 ,

[...] elle est prévue par la loi et qu'elle constitue une mesure qui, dans une société démocratique, est nécessaire à la sécurité nationale, à la sûreté publique, au bien-être économique du pays, à la défense de l'ordre et à la prévention des infractions pénales, à la protection de la santé ou de la morale, ou à la protection des droits et libertés d'autrui.

Pour effectuer un tel contrôle, la Cour vérifie si la mesure prise par l'autorité publique est bien proportionnée par rapport aux buts poursuivis. Le fait qu'une mesure poursuive un but d'intérêt général éminent, comme la protection des espaces naturels, la salubrité et la sécurité publiques, n'est pas en soi suffisant: encore faut-il apprécier l'ensemble des circonstances de l'affaire, et tenir compte en particulier de la vulnérabilité des gens du voyage, du fait qu'ils constituent une minorité ${ }^{27}$. En cassation, tant le juge administratif que le juge judiciaire censurent les décisions des juges du fond qui méconnaissent la grille de lecture établie par la Cour européenne, qu'il s'agisse de décisions de refus de raccordement à l'électricité, ou de demandes d'expulsion de personnes vivant en habitat précaire ou en caravanes ${ }^{28}$.

Ce faisant la Cour contribue à la protection des minorités, déjà protégées au titre de l'article 14 de la Convention.

2. Mais il n'est pas possible de prendre la mesure de l'articulation entre le droit humanitaire européen et le droit de l'urbanisme français si on n'examine pas la façon dont la Cour effectue son contrôle de proportionnalité. Il y a plus de dix ans, Jean-Claude Bonichot écrivait:

[...] la Convention européenne des droits de l'homme ne remet pas en cause les éléments essentiels de notre droit de l'urbanisme, elle joue en revanche, avec la Cour, son rôle premier: mettre les droits fondamentaux à la première place et inciter le législateur comme le juge à la vigilance ${ }^{29}$.

25. Cass. crim., 17 juin 2008, Gérard Dezempte, Bulletin criminel, nº 148, p. 655 : Revue de sciences criminelles, $\mathrm{n}^{\circ} 2$, avril-juin 2009 , p. 380 , note critique C. Mascala; Cass. crim., 21 juin 2011, Alain Tuduri, Bulletin criminel, $\mathrm{n}^{\circ}$ 143, p. 581: Revue de droit immobilier, 2011, p. 507, obs. G. Roujou de Boubée.

26. Cour EDH, GC, 18 janvier 2001, $M^{\text {me }}$ Chapman c. Royaume-Uni, $\mathrm{n}^{\circ} 27238 / 95 ; 17$ octobre 2013, Winterstein c. France, $\mathrm{n}^{\circ} 27013 / 07$.

27. Dans son arrêt Winterstein, la Cour affirme que «la vulnérabilité des Roms et gens du voyage, du fait qu'ils constituent une minorité, implique d'accorder une attention spéciale à leurs besoins et à leur mode de vie propre tant dans le cadre réglementaire valable en matière d'aménagement que lors de la prise de décision dans des cas particuliers [...] ; dans cette mesure, l'article 8 impose donc aux États contractants l'obligation positive de permettre aux Roms et gens du voyage de suivre leur mode de vie» ( $\$ 148$, dernier al.).

28. CE, 15 décembre 2010, $M^{\text {me }}$ Bayer, Recueil Lebon, tables, p. 881: Bulletin de jurisprudence de droit de l'urbanisme, $\mathrm{n}^{\circ}$ 2, 2011, p. 96 , concl. N. Escaut,

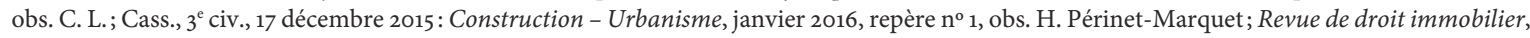
2016, p. 100, obs. P. Soler-Couteaux. Étaient en cause dans ces deux affaires des gens du voyage.

29. J.-C. Bonichot, «Convention européenne des droits de l'homme et droit de l'urbanisme», in Libertés, justice, tolérance. Mélanges Gérard CohenJonathan, Bruxelles, Bruylant, 2004, p. 283; cité par P. Sablière dans la Revue juridique de l'économie publique, $\mathrm{n}^{\circ} 688$, juillet 2011, comm. $^{\circ} 36$. 
Convient-il, dans ces conditions, de considérer, à l'instar de Jean-Claude Bonichot, que l'application jurisprudentielle de la Convention ne conduit qu'à quelques aménagements entre notre droit de l'urbanisme et les droits fondamentaux garantis par ce traité, ou bien, comme le pense Hugues Périnet-Marquet, que le respect des minorités va conduire à «faire trembler le droit de l'urbanisme dans ses fondements mêmes $»^{30}$ ?

L'interprétation des arrêts de la Cour de Strasbourg est parfois délicate dès lors que la Cour entend effectuer un contrôle concret des mesures dont la compatibilité avec la Convention est en cause. Si dans l'arrêt Winterstein de 2013, la Cour conclut à une violation de l'article 8, c'est parce qu'elle constate que la commune a longtemps toléré l'occupation de terrains privés par les gens du voyage, que ceux-ci s'y étaient socialisés, et que le non-respect de la réglementation fixée par le plan d'urbanisme n'apparaissait pas en l'espèce comme d'une importance telle qu'il justifiait une demande d'expulsion en référé. Certes l'appartenance des requérants à une minorité bien identifiée a fortement joué en faveur de la reconnaissance d'une violation de l'article 8 , mais il ne semble pas que cette circonstance ait joué le rôle déterminant que lui attribue Hugues Périnet-Marquet. Celui-ci considère que la Cour de Strasbourg poserait comme principe que toute minorité aurait «un droit à voir respecter son mode de vie et, notamment, son mode de logement ", de telle sorte que, de fil en aiguille, toute règle d'urbanisme fixant un certain type de construction devrait être passée au crible d'un contrôle de proportionnalité entre l'objet de la règle d'un côté, et la préservation d'un mode de vie traditionnel de l'autre. Au final, la jurisprudence de la Cour créerait des discriminations à rebours au détriment de ceux qui ne font pas partie d'une minorité, et donc... de la majorité de la population. Ce serait alors la légitimité même de la règle d'urbanisme qui risquerait d'être remise en cause par le corps social.

Cette notion de discrimination à rebours paraît relever assez largement du fantasme. On aurait tort toutefois de l'écarter d'un revers de main. La protection des minorités ne doit conduire à écarter le respect de la règle d'urbanisme que dans des cas très circonscrits, où, en filigrane, c'est la notion de dignité humaine qui est en jeu. Si tel n'était pas le cas, il y aurait bien un risque de la nature de celui que craint Hugues Périnet-Marquet : toute discipline urbaine serait alors mise en échec.

Les deux faces de la discrimination révèlent en définitive une certaine crise du droit de l'urbanisme, c'est-à-dire sa capacité d'encadrement de la vitalité urbaine, et donc du vivre ensemble. Ce droit participe aussi d'une certaine forme d'impuissance publique. 